\title{
A comparative study of different graft materials used in myringoplasty
}

\author{
B. J. Singh · A. Sengupta - Sudip Kumar Das · D. Ghosh · B. Basak
}

\begin{abstract}
Two hundred twenty cases of unilateral chronic suppurative otitis media (CSOM) with dry central perforation were chosen for this study and myringoplasty were done. Age group ranged from 13 to 48 years. Four types of autogenous tissues were used as graft material. Grafting was done by underlay technique when temporalis fascia, tragal perichondrium, areolar tissue were used as graft material and when fat graft was used the ear lobule fat was placed directly into perforation through transcanal route. Postoperative follow-up was carried out up to 6 months. In this study, it was found that the younger age group has less impairment of hearing and better chance of tympanic membrane perforation closure than the older age group in CSOM with central perforation. Anterior perforations has less impairment of hearing and better result in successful closure of tympanic membrane than posterior perforation group. It was also observed that larger the size of perforation greater is the hearing impairment preoperatively and postoperative hearing gain is also less compared to small perforation. Best hearing improvement occurred using temporalis fascia. Failure occurred may be due to postoperative infection, respiratory tract infection, neglected post-operative advice etc.
\end{abstract}

Keywords Myringoplasty - Chronic suppurative otitis media $(\mathrm{CSOM}) \cdot$ Grafting $\cdot$ Hearing impairment

B. J. Singh ${ }^{1}$ - A. Sengupta ${ }^{2}$ - S. K. $\operatorname{Das}^{2}(\bowtie) \cdot$ D. Ghosh ${ }^{2}$ •

B. Basak $^{2}$

${ }^{1}$ Department of Head \& Neck Oncology, Tata Memorial Cancer

Hospital, Bombay, India

${ }^{2}$ Department of ENT,

IPGME\&R, Kolkata, India

E-mail: sudipdas_ent@rediffmail.com

\section{Introduction}

The repair of the tympanic membrane has been attempted with large variety of synthetic, homologous and autogenous tissue, however temporalis fascia, areolar tissue and perichondrium are used most commonly today. This study was undertaken to compare the easily available autogenous graft materials, i.e. temporalis fascia, tragal perichondrium, areolar tissue and ear lobule fat. The study also compared the result of these grafts in various aspects, the prime interest was the permanent closure of tympanic membrane perforation and hearing restoration in successfully grafted ear.

\section{Aims and objectives}

The present study was undertaken to compare the results of various autogeneous tissues as graft materials for the repair of the tympanic membrane defect. The present study comprised materials which are easily available in adequate amount. These are temporalis fascia, tragal perichondrium, areolar tissue and ear lobule fat. In this study underlay technique was followed in all moderate to large size perforations and tiny perforations were closed by placing fat directly into the perforation through transcanal route.

The primary objectives of this study are (i) to study the graft up in 4 types of autogenous graft materials and (ii) to evaluate the hearing gain of the patient.

\section{Materials and methods}

This study was carried out in Department of ENT, IPGME\&R and SSKM Hospital, Kolkata between May 2005 and April 2006.

All patients were examined clinically and investigations (pure tone audiogram, X-ray of mastoid lateral oblique view, X-ray paranasal sinuses occipitomental view, com- 
plete hemogram, examination of ears under microscope were carried out.

Selection criteria of the patient for this study were:

1. Chronic suppurative otitis media (CSOM) with central perforation, the ear should be dry for at least last 3 months

2. Other ear is normal

3. Pure, conductive type of hearing loss

4. Age above 12 years and below 50 years

5. Except ear disease no other disease condition which could affect the result of the study

In this study 220 subjects were selected (male - 94, female - 126). Autogenous graft materials were used. Temporalis fascia was used in 80 ears, tragal perichondrium used in 20 ears, areolar tissue and ear lobule fat were used in 20 and 100 ears respectively. 208 patients were operated under local anesthesia under sedation. 12 cases were done under general anesthesia. Operations were performed through postaural route (except cases where ear lobule fat was used as graft material). Temporalis fascia and areolar tissue were taken by upward extension of postaural incision. Tragal perichondrium was harvested from the tragus of the same ear. Fat from the ear lobule was harvested by giving $8-10$ $\mathrm{mm}$ horizontal incision on the posterior aspect of ear lobule. While using temporalis fascia, areolar tissue or tragal perichondrium as graft, the approach was postaural and graft was placed beneath the elevated tympanomeatal flap lateral to handle of malleus. When ear lobule fat was used the approach was transcanal route and fat graft placed directly into the perforation. Stitches were removed on the 7th postoperative day. After 3 weeks the ear canal was inspected and position of graft and its vascular status was assessed.
Follow-up was done at 6 weeks, 12 weeks and then at 6 months. Pure tone audiometry was done after 12 weeks in cases with successful closure, and changes in hearing were compared with preoperative audiogram.

\section{Results and analysis}

Age of the subjects in the present study varied from 13 to 48 years. Maximum numbers of subjects were from 21 to 30 years age group (34.55\%) followed by $28.18 \%$ in $31-40$ years age, $24.55 \%$ in $11-20$ year age group and $12.73 \%$ in $41-50$ years age group.

All the perforations were central perforation and they were categorized as 'tiny' ( $<5 \%$ pers tensa involved), 'moderate size' perforation ( $>5 \%$ to $<50 \%$ pers tensa involved) and large ( $>50 \%$ area of pers tensa involved). According to the position of perforation in relation to handle of malleus, the perforations were classified as anterior, posterior and large central perforation.

Investigations were done. In all cases hemogram values were within normal limits. Sclerotic mastoid was found in $162 / 220$ cases and cellular mastoid in 58/220 cases. Maxillary sinus opacity was found in 54/220 cases. Pure tone audiometry done in all cases showed air conduction (AC) threshold range at 22.8-47.7 dB (mean: $32.3 \mathrm{~dB}$ ) and range of bone conduction threshold at $6.6-13 \mathrm{~dB}$ (mean: 8.3 $\mathrm{dB})$. The average air-bone (A-B) gap were in the range of 16.2-41.1 dB (mean: 27.6 dB).

Successful closure of tympanic membrane in 11-20 years age group was $93 \%$ (50/54 cases) with mean hearing gain of $9.3 \mathrm{~dB}$. In 21-30 years age group mean hearing gain was $9.8 \mathrm{~dB}$ and the success rate of tympanic membrane clo-

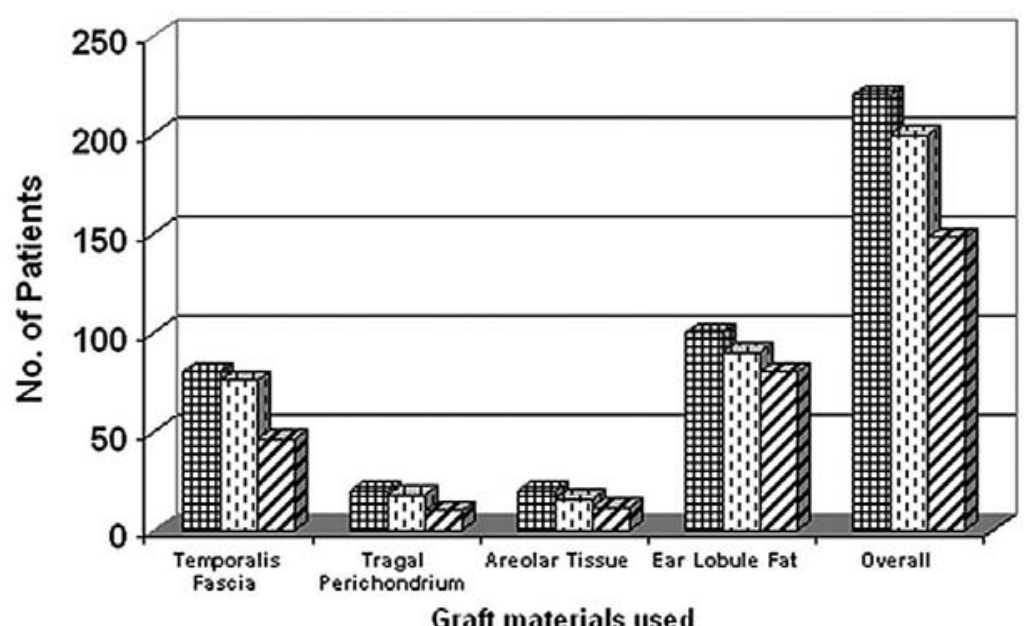

田No. of Cases 口Successful closure $\square$ Successful Hearing

Fig. 1 Results of myringoplasty with respect to graft materials used 
sure was $95 \%$ (74/78) cases. There were $54 / 62$ cases $(87 \%)$ showed successful closure of their perforation in 31-40 years age group followed by $77 \%$ ( $20 / 28$ cases) success rate in 41-50 years age group and mean hearing gains were 8.6 $\mathrm{dB}$ and $8.27 \mathrm{~dB}$, respectively. In $93 \%$ (74/80) cases of anterior type perforation successful closure occurs with mean hearing gain of $7.2 \mathrm{~dB}$, followed by $92 \%$ (44/48) cases of posterior perforation with mean hearing improvement of 7.5 dB. $89 \%$ (82/92) of large central perforation showed successful closure and mean hearing improvement was $9.9 \mathrm{~dB}$.

When comparing the success rate of tympanic membrane closure with different graft materials, temporalis fascia showed best (95\%) closure rate and $9.3 \mathrm{~dB}$ hearing gain, followed by tragal perichondrium $(90 \%$ closure rate and $8.5 \mathrm{~dB}$ hearing improvement) and areolar tissue (80\% closure rate and $8.9 \mathrm{~dB}$ mean hearing improvement). Ear lobule fat was used for closure, of tiny central perforations and it showed $90 \%$ successful closure and $6.8 \mathrm{~dB}$ hearing gain. The over all success rate of tympanic membrane closure was $91 \%$.

When comparing air-bone gap closure using different graft materials temporalis fascia (used in 80 cases) showed good (11-20 dB) A-B gap closure in $63 \%$ cases, fair (21-30 dB) A-B gap closure in $24 \%$ cases and poor (>30 dB) A-B gap result in $13 \%$ cases. Graft failure occurred in 4 cases. Tragal perichondrium used in 20 cases; 10 cases showed good (11-20 dB) A-B gap closure, 6 cases showed fair (21-30 dB) A-B gap closure and 2 cases showed poor ( $>30$ dB A-B gap) result. 20 cases were done using areolar tissue as graft material, among them 12 cases show good (11-20
$\mathrm{dB}$ ) hearing gain, 4 cases showed fair $(21-30 \mathrm{~dB})$ hearing gain and graft failure occurred in 4 cases. Fat graft myringoplasty was done in 100 cases. 16 cases showed excellent $(0-10 \mathrm{~dB})$ hearing gain, 64 cases showed good hearing gain and 10 cases showed fair hearing gain. Graft failure occurred in 10 cases.

To sum up, hearing gain occurred in every case of successful closure of perforation.

\section{Discussion}

In this present study operations were performed in all 220 cases. The mean age was 28.9 years. 94 patients were male and 126 were female. Majority (60\%) of the patients were of rural habitant and rest (40\%) were from urban areas. All of the patients had complaints of discharging ear of varied duration and varied degree of hearing loss. Dry ear for at least 3 months was the main criteria followed [1]. In all the cases both preoperative and postoperative audiometry were performed and results were compared. Postoperative pure tone audiometry was performed only in successful closure cases after 12 weeks follow-up period [2].

In this series maximum successful closure of perforation (95\%) and maximum successful hearing improvement $(84 \%)$ were in the $21-30$ years age group. In $11-20$ years age group successful closure was $93 \%$ and successful hearing was $68 \%$, this is followed by $31-40$ years age group ( $87 \%$ closure rate and $74 \%$ hearing gain) and $41-50$ years age group ( $77 \%$ closure rate and $60 \%$ hearing gain).
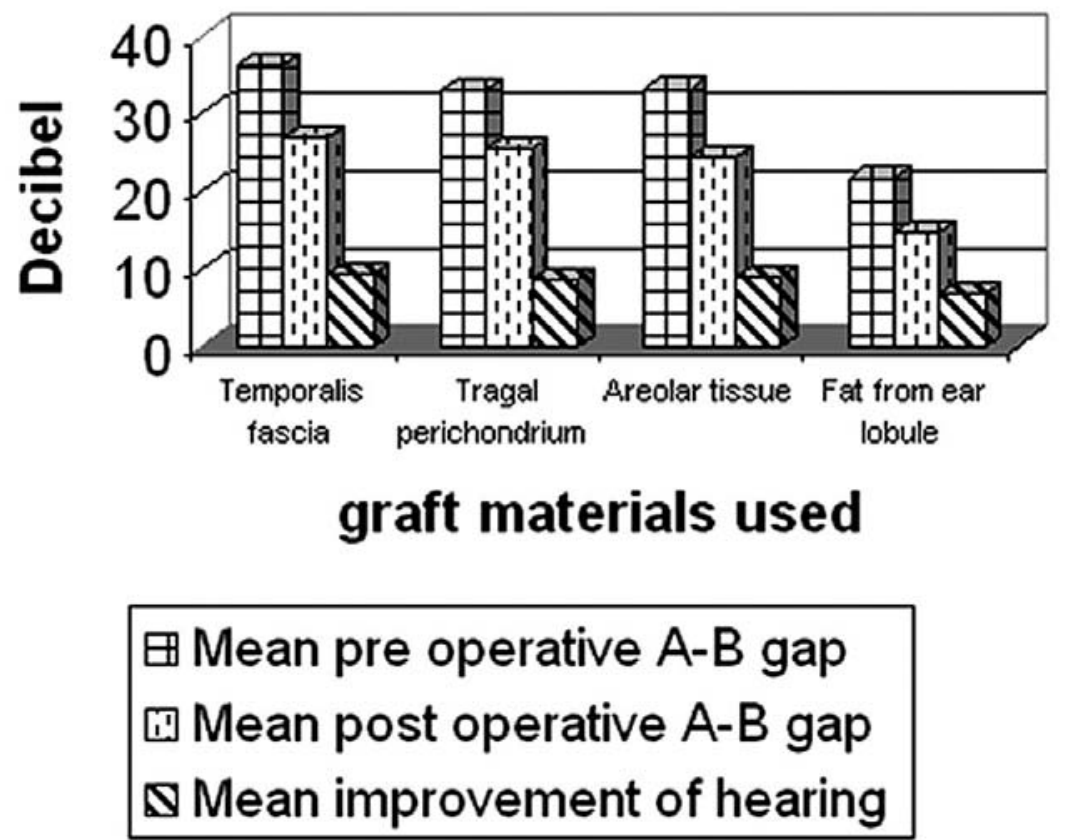

Fig. 2 Decibel wise improvement in A-B gap in different graft materials used 
Graft failure and poor hearing were observed more commonly in older age group where as successful closure and hearing improvement, were universally good in younger age group [3]. So age of the subject affect the overall outcome in myringoplasty operation [4]. Some observers suggested that tympanoplasty surgery can be performed in elderly patients for same indication as for young patients if case selection is proper [5].

In this study successful closure of perforation of $93 \%$ and successful hearing improvement of $84 \%$ occurred in anterior type perforation. For posterior and central perforations the rate of successful closure were $92 \%$ and $89 \%$ and successful hearing gain $82 \%$ and $63 \%$ respectively. So hearing improvement, is better in anterior type perforation than posterior type perforations [6]. It is also observed that successful repair is more in case of small perforation than large perforation. Hearing loss is increased with increased size of perforation [6].

Temporalis fascia is the current gold standard graft material for tympanoplasty. In the present study maximum hearing improvement is achieved with temporalis fascia (mean hearing gain $9.3 \mathrm{~dB}$ ). Temporalis fascia has certain advantages like (i) absence of elastic tissue with no problem of shrinkage makes graft take up better and (ii) structure of temporalis fascia is similar to the missing tunica properia of the drum [7].

In the present study successful closure of tympanic membrane using areolar tissue as graft material is $80 \%$ and successful hearing gain is $75 \%$. The areolar tissue in addition to containing abundant collagen and reticular fibres also houses the fibroblast and macrophages necessary for proper graft incorporation [8].

The time taken for taking tragal perichodrium graft was longer. But no significant difference was noted between fascia and perichondrium graft in hearing gain [9].

Ear lobule fat graft, produced $90 \%$ successful closure and $89 \%$ successful hearing gain. It was particularly used for tiny perforations. Surgical intervention was minimum in these cases. In the present series of 220 patients successful closure seen was $91 \%$ and successful hearing gain occurred in $74 \%$ cases.

Healing of the perforation was complete in most of the cases in 2-3 months except in 4 cases where tragal perichondrium was used and it took about 4 months for healing. Among the 10 cases of graft failure 2 cases with tragal perichondrium and 4 cases each using temporalis fascia and areolar tissue were seen. In 6 cases ( 4 cases of areolar tissue graft and 2 cases of temporalis fascia graft) graft rejection occurred within 6 weeks of postoperative period. In other 4 cases rejection occurred after 10 weeks due to acute otitis media. So early failure might be due to large perforation there by getting poor blood supply and less attachment with rim of perforation margin or may be due to postoperative infection due to poor hygiene and inattentive postoperative advice.

Eight cases of myringoplasty with temporalis fascia developed small perforation after 3-4 months. 4\% among those cases responded to trichloroacetic acid (TCA) cautary while other 4 cases responded to fat graft myringoplasty.

Ten cases of failure occurred in CSOM with tiny perforations when closure was done by ear lobule fat. One week after the operation fat graft was in situ in 76 cases out of 100 cases. In remaining 24 cases fat graft was displaced. In 14 cases perforation decreased in size and healed subsequently with conservative measures. In remaining 10 cases perforation increased in size and those were the actual failure cases. Causes of failure may be infection despite antibiotic coverage, respiratory tract infection producing cough and sneezing leading to graft displacement and continued pond bathing against advice etc.

\section{References}

1. Palva T (1982) Pitfalls in myringoplasty. Acta Otolaryngologica 93:441

2. Mendel L and Kuylen SR (1985) A clinical comparison of the result of two different methods of closing tympanic membrane perforation. J Laryngol Otology 99:339-342

3. Storahan RW, Ward PH, Acquarelli M and Jafek B (1971) Tympanic membrane grafting analysis of materials and techniques. Ann Otorhinolaryngol 80:854-860

4. Hardijk, A Jand and Rietema SJ (1982) Tympanic membrane grafting with fascia, perichondrium and vein. J Laryngol Otology 96:43-47

5. Virtanen H, Palva T and Jauhiainen T (1980) The prognostic value of eustachian tube function measurement in tympanoplasty surgery. Acta Otolaryngologica 90:317-323

6. Yung MW (1983) 'Myringoplasty' hearing gain in relation to perforation site. J Laryngol Otology 97:11-17

7. Lebo CP (1963) Graft selection for tympanoplasty. Ann Otorhinolaryngol 72:40-49

8. Eisenties JF and Hermann BW (2004) Areolar tissue graft in pediatric tympanopalsty - a pilot study. Am J Otolaryngol 25:79-85

9. Gierek T, Slaska-Kaspera A, Majzel K, Klimzak-Golab L (2004). Result of myringoplasty and type I tympanoplasty with the use of fascia, cartilage and perichondrium grafts. Otolaryngology Pol 58:529-533 